\title{
LIGHT CURVE VARIATIONS OF THE OLD DISK ECLIPSING BINARY R CANIS MAJORIS
}

\author{
Y.W. KANG \\ Sejong University \\ kangyw@astro.sejong.ac.kr \\ AND \\ E. F. GUINAN \\ Villanova University \\ guinan@ucis.vill.edu
}

$\mathrm{R} \mathrm{CMa}$ is one of the best observed low mass Algol-type systems, believed to be a semidetached system composed of a nearly spherical FOV primary and a less massive highly distorted K3 IV-V star. Most of the published light curves have asymmetries of one kind or another. Analysis of the individual light curves, dating back to over 75 years, yielded somewhat different orbital solutions depending on the epoch. These inconsistencies are caused by obvious differences in the shape of the light curves obtained at different epochs and by the different models used to find solutions.

A series of light curves of R CMa (obtained at different epochs) have been analyzed by a new version of the Wilson-Devinney computing program to find a homogeneous solution. All parameters were adjusted to fit a light curve of each epoch. Then we found the weighted mean value of each parameter. These mean values were used as initial parameters and the potential and radius of each component were re-adjusted for each epoch to see the variation of radii for over 30 years. Several radial velocity curves were used to find spectroscopic parameters. The photometric parameters were combined with the corresponding spectroscopic parameters to calculate an absolute dimension of R CMa.

Finally, we examined the IUE spectra of $\mathrm{R} \mathrm{CMa}$ for existence of circumstellar gas in the system and evidence of magnetic activity on the cooler component. We also discuss the evolution of the system and possible observable real time evolutionary processes. 